\title{
The implicit nature of the anti-fat bias
}

\author{
HaraldT. Schupp* and Britta Renner \\ Department of Psychology, University of Konstanz, Konstanz, Germany
}

\section{Edited by:}

Patrik Vuilleumier, University Medical Center and University Hospital Geneva, Switzerland

\section{Reviewed by:}

Sylvain Delplanque, University of Geneva, Switzerland

Ulrike Toepel, University and University Hospital Vaud, Switzerland

*Correspondence:

Harald T. Schupp, Department of Psychology, University of Konstanz,

PO Box 36, 78457 Konstanz, Germany. e-mail: harald.schupp@uni-konstanz.de
The stigmatization and discrimination of obese persons is pervasive in almost any domain of living. At the explicit level, obese people are associated with a wide range of negative characteristics. Furthermore, research with the implicit association test revealed the implicit nature of the anti-fat bias. Building upon these findings, the present study used event-related brain potential recordings in order to assess key features of implicit processes. Participants viewed a series of schematic portrayals of anorexic, medium, and obese body shapes and tools. In a passive viewing condition, participants were asked to simply look at the stimuli and, in a distraction condition, participants were asked to detect a specific tool. Viewing obese body images, as compared to medium or anorexic body images, elicited a positive potential shift over fronto-central sites and a relative negative potential over occipito-temporal regions in a time window from 190 to $250 \mathrm{~ms}$. This evaluative brain response to obese body images was similarly pronounced while participants performed a distraction task. Thus, the findings suggest that the anti-fat bias may occur spontaneously, unintentionally, and independent of explicit processing goals. A troublesome picture is emerging in Western cultures suggesting that obese-ism may appear to be as inevitable as a reflex.

Keywords: anti-fat bias, obese-ism, stigmatization, affect, implicit, ERP, EPN

\section{INTRODUCTION}

Almost half of Americans would be willing to give up a year of their life to avoid being fat, and $15 \%$ have reported they would give up 10 or more years of life (Schwartz et al., 2006). This is only one example of the pervasiveness of "obese-ism" in Western cultures, the discrimination and stigmatization associated with obesity. Overweight and obese people face marked social disadvantages in almost any domain of living, including interpersonal relationships, employment, education, and healthcare (Puhl and Brownell, 2001; Puhl and Latner, 2007). Ironically, the social distress induced by obese-ism may worsen or even cause adiposity related disorders (Muennig, 2008). In contrast to widely recognized social stigmata, such as race or gender, there are no legal sanctions in place to protect individuals from obese-ism. This fact is of particular concern as the prevalence of obese-ism has increased in recent years, and the growth is only partly explained by changes in obesity rates (Andreyeva et al., 2008).

Explicit measures consistently reveal that being obese is associated with a wide range of negative characteristics. Obese people are perceived among others as unattractive, unlikable, weak-willed, and alienated from their sexuality (Goodman et al., 1963; Monello and Mayer, 1963; Millman, 1980; Harris et al., 1982; Crandall, 1994). Furthermore, several studies demonstrated that the weight bias is apparent already in early childhood (Puhl and Latner, 2007). Negative attitudes against overweight peers have been observed in children as young as age 3 , and the bias appears to increase from age 4 to 11 (Wardle et al., 1995; Cramer and Steinwert, 1998). However, deliberative or "explicit" obese-ism assessed by self-reports may only represent the tip of the iceberg as the anti-fat bias may have its roots in "implicit" processes.
The implicit nature of the anti-fat bias has been investigated in most studies with the implicit association test (IAT; Greenwald et al., 1998). In this test, automatic associations held toward body weight are assessed through a series of sorting task requiring speeded responses. One task relates to body weight in which stimuli are classified as "thin" or "fat." In the second task, stimuli are categorized according to an evaluative attribute, e.g., "positive" vs. "negative." In the critical conditions, both tasks are to be performed in alternation, while the mapping of the response keys to both tasks is varied. It is assumed that behavioral responses mapping on the same response keys are easier to make when category and attribute task are associated with each other. Consistent with this notion, classifying the categories of (bad + fat) and (good + thin) on the same response keys results in better performance as compared to classifying categories of (bad + thin) and (good + fat) on the response keys. Across studies, a consistent and reliable anti-fat bias has been revealed in college students, health professionals, and recently, in a large online sample ranging in age from 13 to 79 years (Bessenoff and Sherman, 2000; Teachman and Brownell, 2001; Teachman et al., 2003; Wang et al., 2004; Puhl et al., 2005; Ahern and Hetherington, 2006; Schwartz et al., 2006). Revealing the implicit basis of the anti-fat bias is important as it may provide unique insights into the automatic processing of real-life behavior. Specifically, the activation of negative attitudes toward obese individuals may trigger discriminative behavior reflected in non-verbal behaviors such as eye contact, facial expression, and spatial distance. Such immediate negative behaviors may occur in the absence of reflective thinking and even escape awareness providing a constant source of discrimination triggered by the mere sight of an obese person. 
Although performance-based implicit measures (e.g., IAT and priming) provide support for the automatic nature of the anti-fat bias, automaticity is a multifaceted phenomenon, which cannot be comprehensively captured by an all or none approach (Moors and De Houwer, 2006). A core feature of automatic processes is that they operate independent of explicit processing goals (Moors and De Houwer, 2006). However, during the IAT procedure, participants are asked to classify stimuli according to their body weight. Thus, the IAT encompasses a goal-dependent, explicit judgmental task, which raises the question whether obese-ism is elicited predominately through the explicit task demands when viewing the stimuli. Consequently, a stronger test of the notion of automatic obese-ism would be if the bias appears stimulus-driven, i.e., prompted by merely viewing an obese person, independent from explicit intentions to categorize body weight and concurrent processing goals (Moors and De Houwer, 2006). Thus, one goal of the present study was to determine whether the mere sight of an obese person elicits implicit obese-ism. Presentation of "real" persons may be associated with several confounds such as differences in attractiveness, clothing, attire, and familiarity. Similar to research studying facial expressions (Öhman et al., 2001), the use of schematic drawings varying in body weight allows avoiding these problems. As shown in Figure 1, while varying in body weight, other physical differences among exemplars were controlled. Presenting these drawings allowed determining whether body weight is discriminated spontaneously and in the absence of explicit task demands. Emotion research showed that perceptual input triggers a basic affective evaluation (good vs. bad) and corresponding motivational orientation of approach or avoidance (Cacioppo and Berntson, 1994; Lang et al., 1997). Event-related potential (ERP) studies revealed a relatively early and late differential ERP activity associated with the processing of affectively charged stimuli such as viewing pictures of mutilations and gestures of insult, reading negative adjectives or being exposed to clashing moral statements, and images (Schupp et al., 2004, 2007a; Kissler et al., 2007; Stockburger et al., 2009; Van Berkum et al., 2009; Flaisch et al., 2010; Wieser et al., 2010). Specifically, in the time range between 150 and $300 \mathrm{~ms}$, emotional stimuli elicit a relative negative potential over occipito-temporal and a relative positive potential over fronto-central regions followed subsequently by increased late positive potentials (LPP) over centro-parietal regions (i.e., between 300 and 700 ms). Accordingly, the passive viewing condition examines the issue whether obese body drawings elicit early and late ERP responses indicative of affective stimulus evaluation.

A further condition allowed determining whether obese-ism occurs when participants pursue processing goals not associated with body weight and person perception. Goal-independency represents a cardinal feature of implicit processes (Ito and Cacioppo, 2000). Introducing an explicit distraction task accordingly allows examining the issue that obese body shapes activate associative affective network structures irrespective of current task goals. In the distraction task condition, participants had to detect a specific carpenter tool, embedded in a continuous stream of equally probable person and tool stimuli (each shown for $650 \mathrm{~ms}$ ). Accordingly, this condition examined whether obese stimuli elicit early and late differential ERP responses indicative of affective stimulus evaluation independent from concurrent processing goals and unintentionally.
Furthermore, analysis of task performance and ERP responses to the tool drawings were undertaken to assure that participants successfully explored the tool detection task. Previous research revealed that target as compared to non-target stimuli also elicit early and late differential ERP responses in explicit categorization tasks. Specifically, target stimuli elicit a relative negative potential over posterior sensor sites (labeled as Selection Negativity, N2, or Posterior Negativity) followed by a LPP, which is often more specifically referred to as P3b component (Johnson, 1988; Smid et al., 1999; Potts and Tucker, 2001; Codispoti et al., 2006). Replication of these effects provided the necessary precondition for considering the main issue of implicit body stimulus processing.

\section{MATERIALS AND METHODS PARTICIPANTS}

Participants were 27 introductory psychology students ( 14 females) from the University of Konstanz. Participants were between the ages of 20 and 33 years $(M=23.3, \mathrm{SD}=4.1)$ and with the exception of one participant, body mass index was in the range from 19 to 25 $(M=22.7, \mathrm{SD}=3.8)$. Eating habits were assessed by the German adaptation of the Three-Factor Eating Questionnaire (TFEQ; Pudel and Westenhöfer, 1989). Mean scores of the dietary restraint, disinhibition, and hunger scales were $6.4(\mathrm{SD}=4.9), 5.3(\mathrm{SD}=3.1)$, and 6.1 $(\mathrm{SD}=3.2)$, respectively. Participants received course credits toward their research requirements.

\section{STIMULUS MATERIALS}

As illustrated in Figure 1, stimulus materials consisted of schematic drawings of humans and tools.

Schematic body drawings (male and female) were prepared based on the Body Image Assessment Scale (Thompson and Gray, 1995). From the nine body images, three were selected for presentation (the middle and the two most extreme stimuli) and were redrawn to provide a clearly distinct variation in body weight and to allow a comparison of obese body images with both medium and anorexic body images. Previous findings indicate that the vast majority of subjects identified the two extreme drawings as being obese respective anorexic. Specifically, 96.1 and $98 \%$ of the participants rated the male and female thin body drawing as anorexic. Similarly, 88.2 and $90.1 \%$ judged the large body drawing as obese (Thompson and Gray, 1995). In accordance with Thompson and Gray (1995), we refer to these drawings as anorexic, medium, and obese body drawings in the present report. However, it should be noted that the more correct term for "anorexic" would be "very low overall body mass" since it can be caused by multiple reasons such as cancer, AIDS, anorexia nervosa, or other diseases which cannot be inferred from the drawings. Please also note that we used the symptom-related term "anorexic" and not the disease term "anorexia nervosa." However, the more correct term "very low overall body mass" is rarely used in research and previous findings revealed that people identify the drawings as being "anorexic." Therefore, we will use the more brief term "anorexic" in order to be consistent with previous research and to make the text more accessible. Furthermore, as also displayed in Figure 1, six pictures (\#12, $114,196,259,176,199)$ depicting carpenter's tools (i.e., axe, hammer, saw, wrench, pliers, screwdriver) were selected from Snodgrass and Vanderwart (1980). 

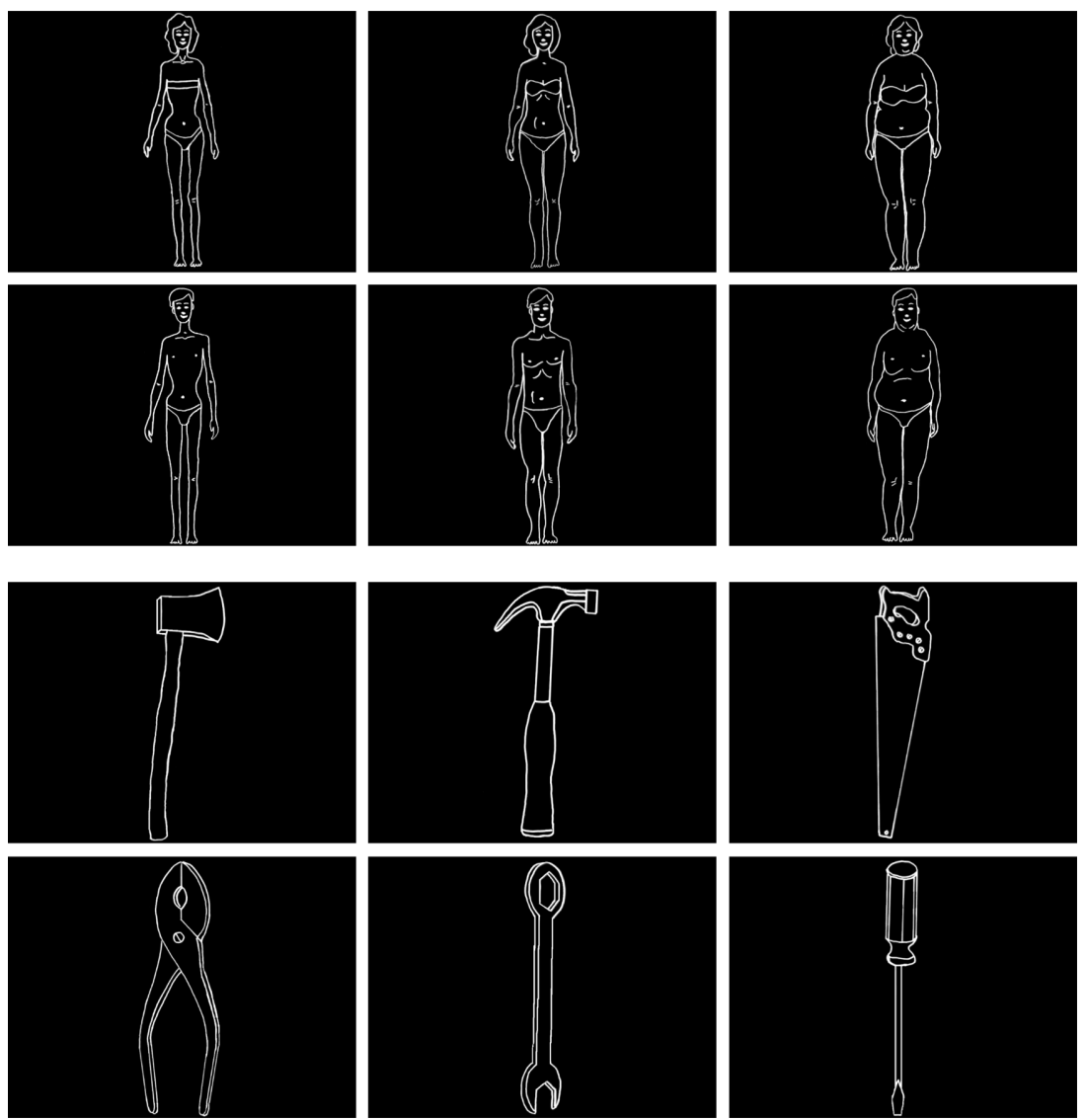

FIGURE 1 | Illustration of the body and tool drawings.

\section{APPARATUS AND STIMULUS PRESENTATION}

The study consisted of two experimental blocks in which the task set for the participants was varied. For assessing spontaneous processing, participants were instructed in the first experimental condition to simply view the pictures. For diverting attention away from body drawings, participants performed an explicit detection task in the second condition. Specifically, they were asked to press a response button as fast and accurate as possible whenever the target stimulus defined as the picture of the hammer tool was shown. Order of experimental conditions was varied across participants.

Both conditions used identical parameters of stimulus presentation. The total of 12 stimuli was presented in a perceptually random order. Several constraints were imposed on the stimulus order to control for sequence effects. Stimuli were never repeated immediately and no more than two repetitions of the same stimulus type (tool and body drawing) were allowed. Furthermore, each participant received his/her own order of picture presentation. Control analysis revealed that transition probabilities of the $\mathrm{N} 1$ stimuli preceding body images were comparable with an average frequency of $9.11(\mathrm{SD}=0.08)$ and $9.10(\mathrm{SD}=0.07)$ for each possible transition in the passive and active task condition, respectively. In addition, a further analysis revealed similar ERP effects as reported in the result section when only body drawings preceded by a tool image were considered in the analysis. To assure high signal-tonoise ratio of ERP measurements, each condition consisted of 1,200 picture presentations, in which each stimulus was shown equally often (100 times). Pictures were presented as continuous stream with no perceivable interstimulus gap and each picture was displayed for $650 \mathrm{~ms}$.

Using Presentation software (Neurobehavioral Systems, Inc., Albany, CA, USA), the pictures were shown on a 22 -inch CRTmonitor ( $60 \mathrm{~Hz}$ refresh rate) located $\sim 124 \mathrm{~cm}$ in front of the participant. Picture presentation lasted for $22 \mathrm{~min}$ with a $\sim 10$-min break between conditions.

\section{SELF-REPORT MEASURES}

Following ERP measurement, participants were asked to rate the body drawings according to their pleasantness and arousal using the Self-Assessment Manikin rating scale (Bradley and Lang, 1994; valence: 1 = most pleasant, $9=$ most unpleasant; arousal: $1=$ least arousing, $9=$ most arousing). Furthermore, attractiveness was rated on a 7 -point Likert scale ( $1=$ unattractive, $7=$ attractive). For statistical analysis, the three self-report measures were entered into a repeated-measures ANOVA with the factor Body Weight (anorexic vs. medium vs. obese) and Stimulus Gender (female vs. male). Furthermore, a median split of the dietary restraint scale of the TFEQ was conducted and as between subject variable in additional 
control ANOVA analysis included. There were no significant effects involving the Restraint Eating factor for any of the three self-report measures.

\section{ERP DATA ACOUISITION AND REDUCTION}

Brain and ocular scalp potential fields were measured with a 256lead geodesic sensor net (GSN 200 v2.0; EGI: Electrical Geodesics, Inc., Eugene, OR, USA), online bandpass filtered from 0.1 to $100 \mathrm{~Hz}$, and sampled at $250 \mathrm{~Hz}$ using Netstation acquisition software and EGI amplifiers. Electrode impedance was kept below $50 \mathrm{k} \Omega$, as recommended for this type of electroencephalogram (EEG) amplifier by EGI guidelines. Data were recorded continuously with the vertex sensor as reference electrode. Continuous EEG data were low-pass filtered at $35 \mathrm{~Hz}$ using a zero-phase forward and reverse digital filter before stimulus synchronized epochs were extracted from $100 \mathrm{~ms}$ before and until $600 \mathrm{~ms}$ after picture onset. Single trial epochs were corrected for vertical and horizontal eye movements using a correlative eye movement algorithm (Miller et al., 1988). The criteria that all sensors are required to be artifact-free in a given trial would result in an unacceptably low number of trials using dense sensor ERP recordings. In order to deal with this problem, data editing and artifact rejection were based on the method for statistically controlling of artifacts (Junghöfer et al., 2000). This procedure (1) detects individual channel artifacts using the recording reference, (2) detects global artifacts using the average reference, (3) replaces artifact-contaminated sensors with spherical interpolation statistically weighted on the basis of all remaining sensors for each individual trial, and (4) computes the variance of the signal across trials to document the stability of the averaged waveform. The rejection of artifact-contaminated trials and sensor epochs relies on the calculation of statistical parameters for the absolute value over time, SD over time, the maximum of the gradient of values over time (first derivative), and the determination of boundaries for each of these three parameters. On average, $22.2 \%(\mathrm{SD}=1.7)$ of the trials showing body drawings were excluded from analysis and there were no significant differences among stimuli and task conditions. Regarding tool drawings, $23.3 \%(\mathrm{SD}=1.7)$ of the trials were excluded with no differences among tool images and task conditions. Data reported here are based on an average reference and baseline-corrected (44 ms) for pre-stimulus ERP activity. Control analysis determined that there were no significant differences in ERP activity in the baseline interval for any of the analysis reported in the Results Section.

\section{ERP ANALYSIS: BODY DRAWINGS}

A two step procedure was used to analyze ERPs to body drawings in the passive and active task condition. The first step was based on visual inspection and exploratory waveform analysis. In single sensor waveform analysis, each time point and sensor was submitted separately to a repeated measure ANOVA including the two within-subject factors "Body Weight" (anorexic vs. medium vs. obese) and "Task" (passive vs. active). Significant effects were thresholded at $P<0.01$ for at least eight continuous data points $(32 \mathrm{~ms}$ ), and two neighboring sensors to provide a conservative guarding against chance findings (Sabbagh and Taylor, 2000). Visual inspection and single sensor waveform analysis revealed that a relatively early differential ERP response was modulated by
Body Weight. Figure 2 illustrates the topographical distribution of $P$-values. Please note, for the purpose of illustration, $P$-values are averaged in this Figure across a time window of $192-248 \mathrm{~ms}$ at which the early effect was most pronounced. Highly significant main effects of Body Weight were observed over fronto-central and occipito-temporal regions. In this time window, neither these nor other regions revealed an interaction of Body Weight by Task Condition. In contrast, neither visual inspection nor single sensor waveform analysis suggested a modulation of the LPP by Body Weight. In a second step, conventional ANOVA and post hoc tests were conducted based on the mean ERP activity averaged across selected sensor sites and time bins.

\section{Early differential ERP activity}

The early differential ERP activity was captured by left and right fronto-central and occipito-temporal sensor clusters in a time window from 192 to $248 \mathrm{~ms}$ at which the effect was most pronounced (see Figure 2). Repeated-measures ANOVAs were calculated including the factors Body Weight (anorexic vs. medium vs. obese), Stimulus Gender (female vs. male), Task (active vs. passive), Location (fronto-central vs. occipito-temporal), and Laterality (left vs. right).

\section{Late differential ERP activity}

To provide a formal test, the LPP was also submitted to ANOVA analysis. Based on previous research, mean ERP activity was calculated in left and right centro-parietal sensor clusters and four time bins between 400 and 588 ms. Repeated-measures ANOVAs were

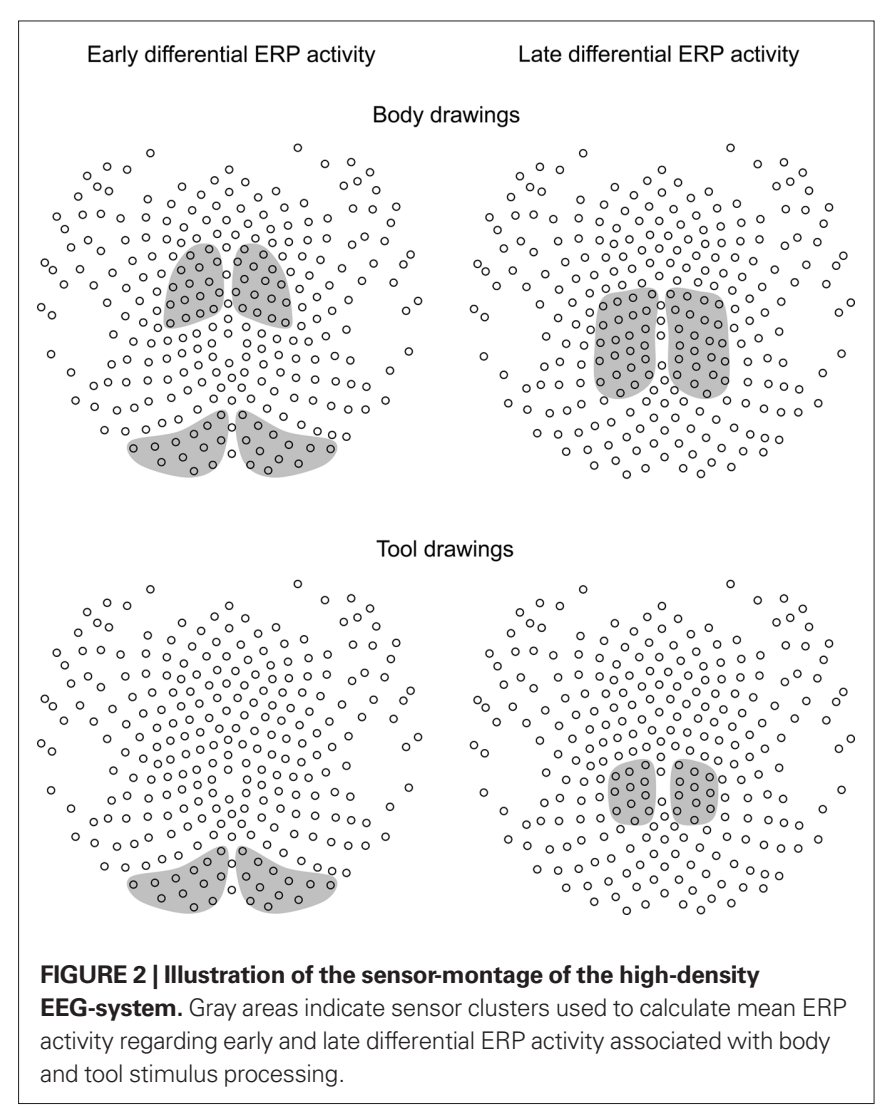


calculated including the factors Body Weight, Stimulus Gender, Task, Laterality, and Time (400-444 vs. $448-492$ vs. $496-540$ vs. 544-588 ms).

For exploratory reasons, initial analysis of early and late ERP activity included also the factor "Participant Gender." As there were no significant effects, this factor was dropped from consideration in the main analyses.

When appropriate, the Greenhouse-Geisser procedure was used to correct for violations of sphericity.

\section{ERP ANALYSIS: TOOL DRAWINGS}

Similarly, a two step procedure was used to explore ERP activity elicited by the tool drawings. First visual inspection revealed that target stimuli in the active task condition were associated with early and late differential ERP activity, replicating previous research (Codispoti et al., 2006). Converging evidence was provided by the single sensor waveform analysis including factors of Task (active vs. passive) and Stimuli (non-target vs. target). Regarding the early differential ERP activity, significant interactions of Task and Stimuli were observed over occipito-temporal and central regions, most pronounced between 200 and 300 ms. Regarding late differential ERP activity, the hallmark finding of increased P3bs to target stimuli resulted in significant interactions of Task and Stimuli over centro-parietal regions, most apparent between 400 and $600 \mathrm{~ms}$. In a second step, both ERP components were submitted to repeatedmeasures ANOVA including factors of Task, Stimuli, and Laterality. The early differential ERP activity was assessed as mean ERP activity in left and right occipito-temporal cluster in a time window from 200 to $300 \mathrm{~ms}$. The late differential ERP activity was assessed as mean ERP activity in left and right centro-parietal clusters across a time window from 400 to $600 \mathrm{~ms}$ (see Figure 2).

\section{BEHAVIORAL PERFORMANCE IN THE TOOL DETECTION TASK}

Behavioral performance was assessed by calculating the $d^{\prime}$ score (Green and Swets, 1966). This measure is based on the difference in $z$-converted measures of hits and false alarms. Positive $d^{\prime}$ scores indicate that participants performed the task as instructed.

\section{RESULTS}

\section{SELF-REPORT RATINGS}

Attractiveness, valence, and arousal ratings of the anorexic, medium, and obese body drawing are illustrated in Figure 3. A significant main effect of the factor Body Weight was observed for attractiveness, $F(2,48)=59.6, P<0.0001$, indicating a decrease in perceived attractiveness from medium to anorexic to obese body images, $t \mathrm{~s}(24)>4.4, P<0.001$. Furthermore, this main effect was qualified by a significant interaction of Body Weight by Stimulus Gender, $F(2,48)=4.9, P<0.05$. This interaction was due to differences in perceived attractiveness for anorexic body images. The female anorexic body image was perceived as more attractive than the respective male body silhouette, $t(24)=4.7, P<0.0001$. Furthermore, female drawings were perceived generally as being more attractive compared to male silhouettes, Stimulus Gender, $F(1,24)=23.3, P<0.0001$.

A similar pattern of results was observed for valence ratings with significant main effects for Body Weight, $F(2,48)=15.6, P<0.0001$, Stimulus Gender, $F(1,24)=13.5, P<0.0001$, and a significant interaction of Body Weight by Stimulus Gender, $F(2,48)=8.4, P<0.01$. For female drawings, obese stimuli were rated as being more unpleasant than medium or anorexic body images, $t s(24)>3.8, P<0.001$. For male drawings, obese and medium stimuli were rated as being more unpleasant than anorexic body images, $t \mathrm{~s}(24)>4.3, P<0.001$. Again, anorexic female drawings were rated as being more pleasant compared to the respective male anorexic silhouette, $t(24)=4.7, P<0.001$.

No significant effects were observed for arousal ratings, $F s<2.4$.

\section{ERP ANALYSES: BODY IMAGE PROCESSING Early differential ERP activity}

The present study obtained evidence for the differential processing of obese body drawings. As illustrated in Figure 3, the encoding of obese silhouettes resulted in a positive shift in the ERP waveform over left and right hemispheric fronto-central cluster of sensors and a relative negative shift over left and right hemispheric occipitotemporal cluster of sensors. The early differential ERP activity is further highlighted in Figure 4 by subtracting the waveforms to obese images from those evoked during viewing of anorexic and medium body weight images. Considering the temporal information displayed in the Figures 1 and 2, the effect of enlarged
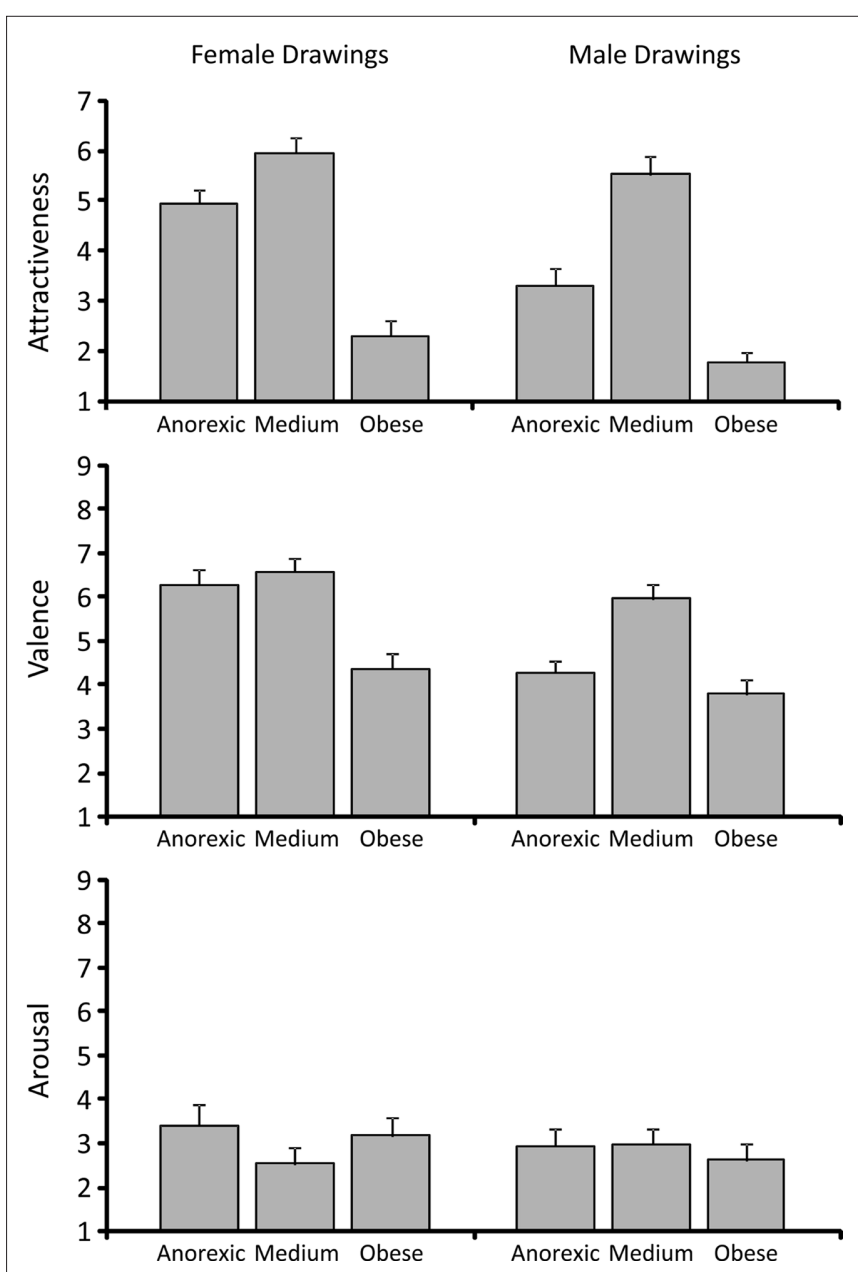

FIGURE 3 | Mean ratings of attractiveness, valence, and arousal of the body drawings, separately for female and male body drawings (left and right panels). 


\section{A \\ Electrodewise Statistics (192-248 ms)}

Main effect:

Bodyweight

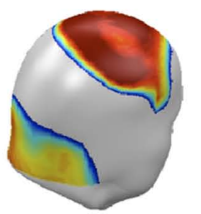

B Passive Viewing
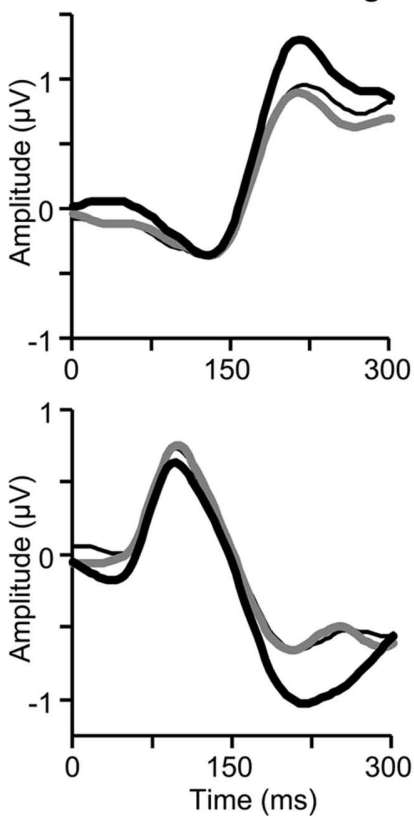

Interaction:

Bodyweight times Task

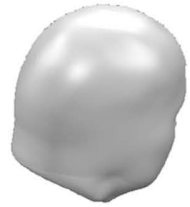

0.001

0.05

0.1

$P$-value

Detection Task
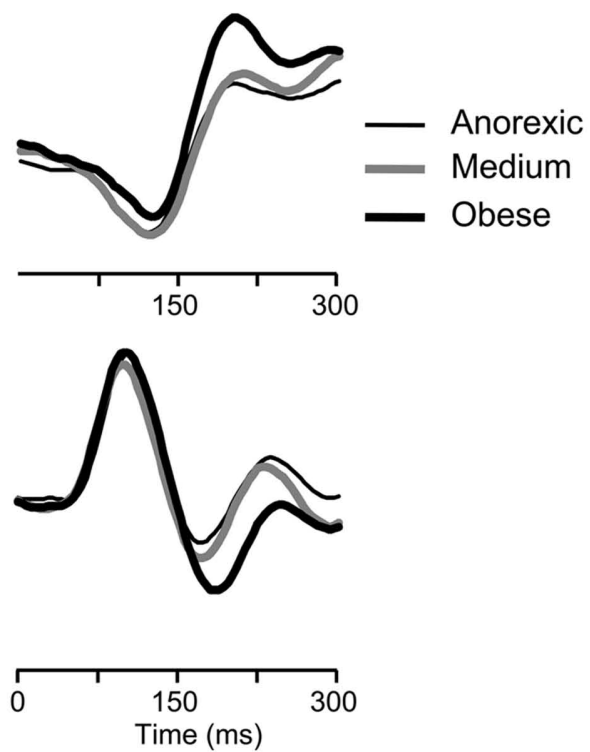

C

\section{ERP Difference Maps (192-248 ms)}

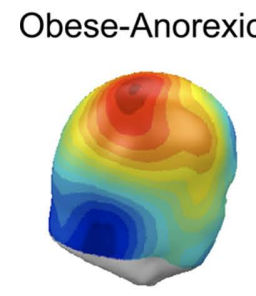

Passive Viewing

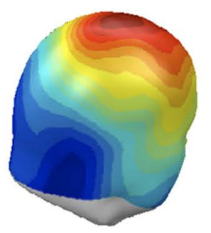

FIGURE 4 | (A) The outcome of the point-by-point waveform ANOVA is illustrated in terms of $P$-values, averaged for the purpose of illustration across the time window from 192 to $248 \mathrm{~ms}$. (B) Illustration of the ERP

waveforms to anorexic, medium, and obese body drawings during passive
Obese-Anorexic Obese-Medium
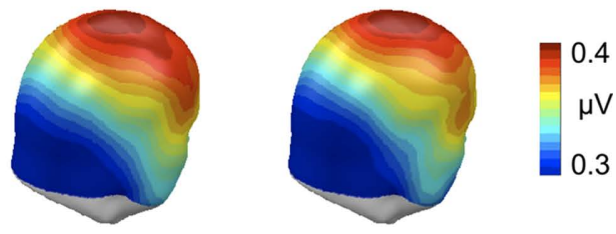

Detection Task

viewing and active task conditions for representative right central (\#132) and occipito-temporal (\#175) sensors. (C) Illustration of the topographical distribution of the original scalp potentials. A back view of a model head is displayed. occipito-temporal negativity for pleasant and unpleasant pictures was maximally pronounced between 192 and $248 \mathrm{~ms}$ after stimulus onset. As expected, the overall analysis revealed a significant interaction of Body Weight and Location, $F(2,52)=11.0, P<0.0001$. Accordingly, separate analyses were conducted for the frontocentral and occipito-temporal clusters.
Fronto-central clusters. Over fronto-central regions, ERP activity varied as a function of Body Weight, $F(2,52)=11.8, P<0.0001$. Post hoc tests revealed an increased positive potential for the obese as compared to both, the medium, $t(26)=4.6, P<0.001$, and the anorexic body image, $t(26)=3.8, P<0.001)$. Furthermore, amplitudes elicited by the medium and anorexic body drawings 
were not significantly different, $t(26)=0.1$, ns. For exploratory reasons, passive and active task conditions were analyzed separately to confirm that obese images were differentially processed in both task conditions. Separate analysis of the passive and active task conditions revealed in both cases significant main effects for the factor Body Weight, $F(2,52)=5.4$ and 8.7, $P<0.01$, respectively. During passive viewing, obese stimuli elicited a significantly larger positive potential compared to anorexic, $t(26)=2.5, P<0.05$, and medium body weight stimuli, $t(26)>3.1, P<0.01$. Similarly, obese stimuli elicited larger positive potentials as compared to anorexic and medium weight stimuli in the active task condition, $t(26)=3.3$ and 4.0, $P<0.01$. Anorexic and medium body images were not significantly different in either of the two conditions, $t(26)<0.07$.

The early ERP activity was furthermore modulated by the Task Condition, $F(1,26)=9.9, P<0.01$, with increased positivity during the passive viewing condition as compared to the tool detection task.

Occipito-temporal clusters. Over occipito-temporal regions, the early ERP activity significantly varied as a function of Body Weight, $F(2,52)=8.3, P<0.01$. Post hoc tests revealed an increased negative potential for the obese as compared to both, the medium, $t(26)=3.6, P<0.001$, and the anorexic body image, $t(26)=3.1$, $P<0.01$. Furthermore, amplitudes elicited by the medium and anorexic body drawings were not significantly different, $t(26)=0.9$, ns. Again, for exploratory reasons, both task conditions were analyzed separately to support the notion of the differential processing of obese images. Analysis of the passive and active task conditions revealed for both conditions significant main effects of Body Weight, $F(2,52)=4.5$ and 5.2, $P<0.05$, respectively. In both conditions, obese stimuli elicited a more negative potential as compared to anorexic and medium body weight images, $t \mathrm{~s}(26)>2.4$, $P<0.025$, while anorexic and medium images were not significantly different from each other, $t(26)=1.0$.

In addition, the early ERP activity was modulated by Task Condition, $F(1,26)=10.5, P<0.01$, with increased negativity during the passive viewing condition as compared to the tool detection task.

\section{Late differential ERP activity}

As shown in Figure 5, LPP were similar for anorexic, medium, and obese body drawings. ANOVA analysis revealed no main effect or interaction involving Body Weight, Fs $<1.9$.

\section{ERP ANALYSES: TOOL IMAGE PROCESSING}

Figure 6 illustrates early and late differential ERP activity associated with the tool detection task. As can be seen, target stimuli elicited a relative negative potential over occipito-temporal sensor sites which was most pronounced between 200 and $300 \mathrm{~ms}$ followed by a pronounced P3b component over centro-parietal sensor sites.

\section{Early differential ERP activity}

A significant interaction of Task by Stimuli was observed for the early differential ERP activity, $F(1,26)=28.6, P<0.0001$, which was followed up by separate analyses of passive and active task conditions. As expected, in the active task condition, target $(M=-1.15 \mu \mathrm{V})$ as compared to non-target $(M=-0.08 \mu \mathrm{V})$ stimulus processing was associated with a relative negative potential over occipitotemporal sensor sites, $F(1,26)=37.1, P<0.0001$. In the passive condition, target stimuli were associated with a somewhat more negative potential as compared to non-target stimuli $(M=-0.53$ vs. $M=0.14 \mu \mathrm{V}), F(1,26)=3.6, P=0.07$. Further analysis determined that participants performing the tool task as first condition showed a sustained effect of task relevance. Specifically, target as compared to non-target stimuli elicited a larger negative potential, $F(1,13)=5.7, P<0.05$. There was no significant difference in processing target and non-target stimuli in participants in which passive viewing was the first condition, $F(1,12)=0.3$, ns.

\section{Late differential ERP activity}

Similarly, a significant interaction of Task by Stimuli was observed for the late differential ERP activity, $F(1,26)=55.5, P<0.0001$. As expected, enlarged $\mathrm{P} 3 \mathrm{~b}$ potentials were observed for target compared to non-target stimuli $(M=3.29$ vs. $M=0.37 \mu \mathrm{V})$ in the active task condition, $F(1,26)=75.4, P<0.0001$. In the passive condition, target stimuli also elicited larger positive potential shift as compared to non-target stimuli $(M=0.31$ vs. $M=0.03 \mu \mathrm{V})$, $F(1,26)=8.4, P<0.01$. This effect was specific to participants performing the active task as first condition, $F(13)=7.8, P<0.25$, and not observed when passive viewing was the first condition, $F(1,12)=1.4$, ns.

\section{TOOL DETECTION TASK: BEHAVIORAL RESULTS}

Performance in the primary tool detection task was captured by the $d^{\prime}$ measure. Behavioral performance in the active task condition was very high $\left(d^{\prime}=4.0, \mathrm{SD}=1.3\right)$ and significantly different from chance performance, $t(23)=15.0, P<0001$.

\section{DISCUSSION}

Using event-related brain potential recordings, the main aim of the study was to investigate the implicit nature of the anti-fat bias. Two experimental conditions investigated core features of automaticity of processing such as being unintentional, goal-independent, spontaneous, stimulus-driven, and fast. The results suggested that a snapshot of an obese person was sufficient for triggering an implicit affective evaluation. A troublesome picture is emerging suggesting that obese-ism may appear to be as inevitable as a reflex in western cultures.

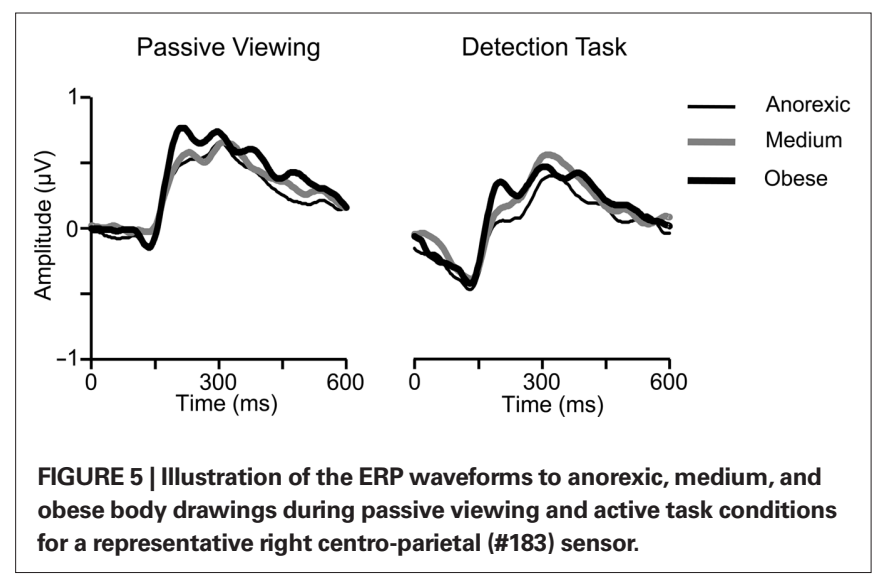




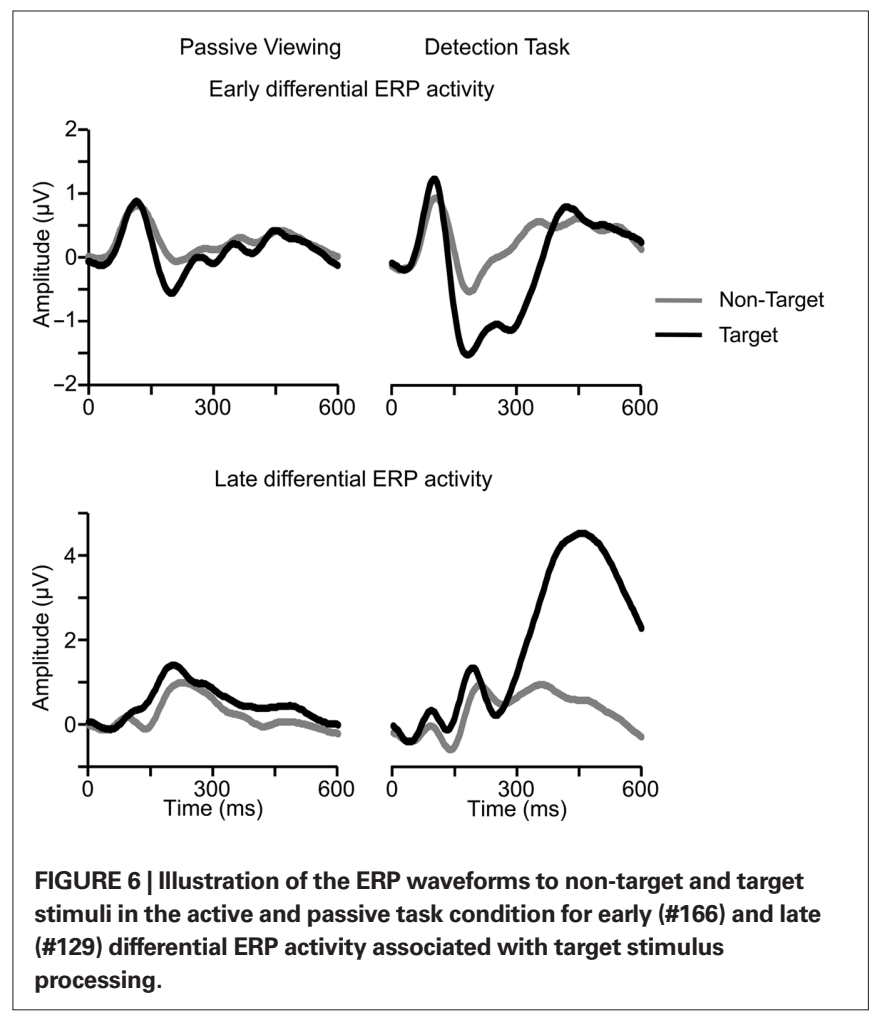

\section{AFFECT AND OBESE BODY SHAPE}

The present study revealed that portrayals of obesity elicited the brain correlate of affective stimulus evaluation. Specifically, an early differential brain activity, maximally pronounced between 192 and $248 \mathrm{~ms}$, differentiated obese from both anorexic and medium body weight images. The effect appeared as a positive potential shift over fronto-central sensor sites and a relative negative potential shift over occipito-temporal regions. These findings are strikingly similar to previous findings regarding an early differential ERP activity associated with the processing of emotionally significant stimuli such as emotional pictures, gestures, faces, and words (Schupp et al., 2004, 2007a; Kissler et al., 2007; Flaisch et al., 2010; Wieser et al., 2010). Accordingly, the perception of obese body weight images elicited the brain signature of affective stimulus evaluation.

However, previous research revealed that both pleasant and unpleasant emotionally significant stimuli modulate this early differential ERP component. Thus, rather than reflecting motivational orientation of approach or avoidance, the early ERP component is presumed to reflect the increased intrinsic stimulus significance of stimuli (Schupp et al., 2006). Tagging obese stimuli relatively early in the process of stimulus categorization is in all likelihood related to negative affect. Specifically, obese stimuli received not only unfavorable ratings at the explicit level in the present study, previous research relying on behavioral paradigms such as the IAT and priming revealed a strong negative bias at the implicit level (Bessenoff and Sherman, 2000; Teachman and Brownell, 2001; Puhl et al., 2005; Schwartz et al., 2006). Collectively, the current and previous findings suggest that obese body shape is encoded in neural networks containing links to the aversive motivational system. Activation of these networks appears stimulus-driven and spontaneously, resulting in a relative early discriminative brain response to obese stimuli.

In contrast to the early differential ERP activity, obese stimuli were not eliciting an increased LPP. Previous research revealed increased LPP amplitudes to a variety of emotionally significant stimuli establishing this component as further reliable indicator of affect processing (Schupp et al., 2006). However, while the LPP is consistently observed in studies investigating stimuli of high emotional intensity, the findings are less robust with regard to stimuli of comparably lower emotional intensity. For instance, in research with emotional words and emotional symbolic gestures, the LPP effect was much less pronounced as observed in research presenting emotionally evocative pictures such as erotica or mutilations (Schupp et al., 2006; Herbert et al., 2008; Flaisch et al., 2010). Based on these findings, emotional intensity of the schematic stimuli may be too low to elicit a reliable LPP effect. Increasing the emotional intensity of the stimulus materials by showing "real" pictures of obesity may accordingly elicit a LPP modulation.

\section{FEATURES OF AUTOMATICITY}

Contemporary conceptions of automaticity share the assumption of defining core features, which need to be investigated separately. It is furthermore widely assumed that the presence of processing features such being unintentional, stimulus-driven, goal-independent, efficient, and fast represent important facets of automaticity (Bargh, 1997; Moors and De Houwer, 2006). Previous research relying on performance-based measures of the implicit anti-fat bias was limited with regard to features of goal-dependency and intentionality. In the IAT procedure, participants are asked to evaluate the weight-related stimuli into dichotomous categories of being anorexic or fat. Thus, consideration of body weight is explicitly task-relevant providing a mindset in which corresponding neural networks need to be actively engaged to solve the task. The passive viewing condition allowed determining the stimulusdriven and spontaneous appearance of an anti-fat bias when participants viewed the stimulus materials without any explicit task demands. A stronger test was provided in the active distraction task condition, in which participants had to detect a specified tool image interspersed in the rapid and continuous stream of pictures. Importantly, target stimuli revealed the expected finding of increased posterior negativity followed by a P3b wave. Thus, behavioral and ERP findings support the notion that participants had a mindset diverting attention away from the body images. Findings across both conditions converged and revealed an early differential ERP activity specifically to obese stimuli supporting the notion that this brain response occurs spontaneously, unintentionally, and independent from current processing goals. Suggesting that the anti-fat bias shows features of goal-independency and un-intentionality has implications regarding the elicitation and occurrence of the bias. The mere sight of an obese person may trigger the anti-fat bias and associated discriminative behaviors. However, this hypothesis rests on the assumption that the early ERP activity reflects the stimulus-driven activation of memories, which is also involved in the organization of spontaneous behaviors (Gawronski et al., 2007). Future research is needed to conclusively examine this hypothesis. 
The relatively early latency of the differential ERP activity (i.e., $\sim 190 \mathrm{~ms}$ ) relates to a further defining feature of automaticity, namely speed and efficiency of processing (Lieberman, 2000; Evans, 2008). A history of experiential learning may provide the basis for relating differences in body weight to personality characteristics, as has been suggested with regard to trustworthiness and competence (Todorov et al., 2005; Bar et al., 2006). Previous research regarding the explicit categorization of higher-order stimulus categories refer to differential ERP activity emerging around $150 \mathrm{~ms}$ after stimulus onset as ultra-rapid categorizations (Thorpe et al., 1996). According to this reasoning, the present findings support the notion regarding the features of efficiency and speed of the implicit anti-fat bias.

A potential limitation of the present findings is that participants may have exerted voluntary top-down control mechanism in processing the body drawings. However, the present findings cannot be explained by cognitive control mechanism operating when obese images are consciously perceived. Strategic control is estimated to require several hundred milliseconds (Neely, 1977), thus taking too long to modulate the early differential ERP activity. An alternative assumption is that participants intentionally directed their attentional focus to body images. However, previous research revealed that paying attention to emotional and neutral stimuli elicited effects associated with task relevance and intrinsic stimulus significance which were independent from each other (Schupp et al., 2007a; Ferrari et al., 2008). Thus, paying attention to the body images does not provide an explanation for the effects specific to obese drawings. Finally, participants may have voluntarily paid attention specifically to obese drawings. However, such a scenario seems unlikely given the nature of the active task condition, i.e., rapid and continuous serial presentation, familiarity with the stimulus materials after the first few repetitions, and performance of a distraction task. Furthermore, the notion that participants voluntarily devoted attention to obese stimuli would have predicted a pronounced $\mathrm{P} 3 \mathrm{~b}$ component to obese stimuli (Johnson, 1988). Inconsistent with this reasoning, only the taskrelevant tool target as compared to non-target stimuli elicited a pronounced $\mathrm{P} 3 \mathrm{~b}$ component. One strategy to circumvent voluntary top-down control mechanisms is to present the distraction task concurrently with the body drawings. This procedure would investigate a further facet of automaticity, i.e., whether the process is dependent on the availability of processing resources. However, there is some suggestion that affective stimulus evaluation as indicated by the early differential ERP activity is dependent rather than independent from the availability of processing resources (Schupp et al., 2007b).

\section{FIRST IMPRESSIONS OF OTHER PEOPLE}

The current findings also extend previous research examining first impressions of other people. This line of research revealed that a picture of a person provides a rich array of information and that people are remarkably efficient about forming impressions of other people regarding socially relevant dimensions such as competence, valence, attractiveness, and competence (Todorov et al., 2008). The self-report findings reveal that body shape is an important cue for first impressions. Specifically, the prototypical shape of obesity was perceived as less attractive and less positive compared to medium and anorexic portrayals. Furthermore, research about first impressions revealed features of automaticity, which appear strikingly similar to the current ERP findings. Specifically, short exposures of stimuli are sufficient to allow judgments of trustworthiness and competence, similar to evaluations based on longer stimulus presentations (Bar et al., 2006; Willis and Todorov, 2006). In addition, impressions about other persons occur spontaneously and even when people are engaged in a distractor task (Todorov and Uleman, 2003). Most related to the present study, a recent functional magnetic resonance imaging study revealed the engagement of an interrelated neural network comprising the amygdala, cingulate cortex, fusiform cortex, and lateral prefrontal cortex in processing negative social stigma cues during implicit and explicit task conditions (Krendl et al., 2006). Taken together, body weight may provide a highly relevant piece of information, which is used to form impressions of other people. Future studies may determine the amplification or amelioration of the anti-fat bias as a function of first impression cues such as facial attractiveness, trustworthiness, and competence.

\section{STRENGTH AND LIMITATIONS}

Similar to previous research studying emotional facial expressions (Öhman et al., 2001), the present study assessed the anti-fat bias by using schematic stimuli varying in terms of prototypical features of body weight. This approach seems particularly relevant as an item-analysis of complex natural scenes revealed that nonemotional features suppress the early and enhance the late differential ERP activity to negative pictures (Wiens et al., 2011). Thus, the strength of using schematic drawings is that the current findings are unlikely to be secondary to differences in non-emotional features. Across the three body weight categories, the stimulus materials were controlled for differences in facial expression, posture, height, clothing, attire, which have been shown to provide the basis for first impressions of other people. While the stimuli were highly controlled in many respects, the issue arises whether the findings reflect differences in body weight or simple differences in stimulus size. The hypothesis of simple size differences is expected to result in a liner relationship of ERP activity and stimulus size. Given that there were no differences among anorexic and medium weight stimuli, the pattern of finding seems in favor of the notion of the anti-fat bias hypothesis.

The current as well as most previous research has been conducted in Western cultures, which evince a pronounced anti-fat bias. However, attitudes toward obesity vary across culture and time as revealed by anthropological records (Powdermaker, 1960). The selective processing of obese stimuli as revealed in this ERP study is presumably only observed in cultures evincing an anti-fat bias. Examining the anti-fat bias from a cross-cultural perspective might accordingly reveal the effects of the socio-cultural transmission of the anti-fat bias.

\section{ACKNOWLEDGMENTS}

We thank Anna Artinyan, Ines Krug, and Tobias Flaisch for their assistance in data collection and analysis. This work was supported in part by grants from the German Research Foundation (Schu 1074/11-2) and the Federal Ministry of Education and Research (BMBF Grant 0315671, granted to Britta Renner and Harald Schupp). 


\section{REFERENCES}

Ahern, A. L., and Hetherington, M. M. (2006). The thin ideal and body image: an experimental study of implicit attitudes. Psychol. Addict. Behav. 20, 338-342.

Andreyeva, T., Puhl, R. M., and Brownell, K. D. (2008). Changes in perceived weight discrimination among Americans, 1995-1996 through 20042006. Obesity 16, 1129-1134.

Bar, M., Neta, M., and Linz, H. (2006). Very first impressions. Emotion 6, 269-278.

Bargh, J. A. (1997). "The automaticity of everyday life," in The Automaticity of Everyday Life: Advances in Social Cognition, ed. J. R. S. Wyer (Mahwah, NJ: Erlbaum), 1-61.

Bessenoff, G. R., and Sherman, J. W. (2000). Automatic and controlled components of prejudice toward fat people: evaluation versus stereotype activation. Soc. Cogn. 18, 329-353.

Bradley, M. M., and Lang, P. J. (1994). Measuring emotion: the selfassessment manikin and the semantic differential. J. Behav. Ther. Exp. Psychiatry 25, 49-59.

Cacioppo,J.T., and Berntson, G. G. (1994). Relationship between attitudes and evaluative space - a critical-review, with emphasis on the separability of positive and negative substrates. Psychol. Bull. 115, 401-423.

Codispoti, M., Ferrari, V., Junghofer, M., and Schupp, H. T. (2006). The categorization of natural scenes: brain attention networks revealed by dense sensor ERPs. Neuroimage 32, 583-591.

Cramer, P., and Steinwert, T. (1998). Thin is good, fat is bad: how early does it begin? J. Appl. Dev. Psychol. 19, 429-451.

Crandall, C. S. (1994). Prejudice against fat people-ideology and self-interest. J. Pers. Soc. Psychol. 66, 882-894.

Evans, J. S. (2008). Dual-processing accounts of reasoning, judgment, and social cognition. Ann. Rev. Psychol. 59, 255-278.

Ferrari, V., Codispoti, M., Cardinale, R., and Bradley, M. M. (2008). Directed and motivated attention during processing of natural scenes. J. Cogn. Neurosci. 20, 1753-1761.

Flaisch, T., Hacker, F., Renner, B., and Schupp, H. T. (2010). Emotion and the processing of symbolic gestures: an event-related brain potential study. Soc. Cogn. Affect. Neurosci. doi: 10.1093/scan/nsq022. [Epub ahead of print].

Gawronski, B., LeBel, E. P., and Peters, K. R. (2007). What do implicit measures tell us?: scrutinizing the validity of three common assumptions. Perspect. Psychol. Sci. 2, 181-193.
Goodman, N., Richardson, S. A., Dornbusch, S. M., and Hastorf, A. H. (1963). Variant reactions to physical-disabilities. Am. Sociol. Rev. 28, 429-435.

Green, D. M., and Swets, J.A. (1966). Signal Detection Theory and Psychophysics. New York: Wiley.

Greenwald, A. G., McGhee, D. E., and Schwartz, J. L. K. (1998). Measuring individual differences in implicit cognition: the implicit association test. J. Pers. Soc. Psychol. 74, 1464-1480.

Harris, M. B., Harris, R. J., and Bochner, S. (1982). Fat, 4-eyed, and female-stereotypes of obesity, glasses, and gender. J. Appl. Soc. Psychol. 12, 503-516.

Herbert, C., Junghofer, M., and Kissler, J. (2008). Event related potentials to emotional adjectives during reading. Psychophysiology 45, 487-498.

Ito, T. A., and Cacioppo, J. T. (2000). Electrophysiological evidence of implicit and explicit categorization processes. J. Exp. Soc. Psychol. 36, 660-675.

Johnson, R. J. (1988). The amplitude of the P300 component of the eventrelated potential. Adv. Psychophysiol. 3, 69-137.

Junghöfer, M., Elbert, T., Tucker, D. M., and Rockstroh, B. (2000). Statistical control of artifacts in dense array EEG/MEG studies. Psychophysiology 37, 523-532.

Kissler, J., Herbert, C., Peyk, P., and Junghofer, M. (2007). Buzzwords: early cortical responses to emotional words during reading. Psychol. Sci. 18, 475-480.

Krendl, A. C., Macrae, C. N., Kelley, W. M., Fugelsang, J. A., and Heatherton, T. F. (2006). The good, the bad, and the ugly: an fMRI investigation of the functional anatomic correlates of stigma. Soc. Neurosci. 1, 5-15.

Lang, P. J., Bradley, M. M., and Cuthbert, B. N. (1997). "Motivated attention: affect, activation, and action," in Attention and Emotion: Sensory and Motivational Processes, eds P. J. Lang, R. F. Simons, and M. Balaban (Mahwah, NJ: Erlbaum), 97-135.

Lieberman, M. D. (2000). Intuition: a social cognitive neuroscience approach. Psychol. Bull. 126, 109-137.

Miller, A. M., Gratton, G., and Yee, C. M. (1988). Generalized implementation of an eye movement correction procedure. Psychophysiology 25 , 241-243.

Millman, M. (1980). Such a Pretty Face: Being Fat in America. New York: Norton.
Monello, L. F., and Mayer, J. (1963). Obese adolescent girls - an unrecognized minority group. Am. J. Clin. Nutr. 13 35-39.

Moors, A., and De Houwer, J. (2006) Automaticity: a theoretical and conceptual analysis. Psychol. Bull. 132, 297-326.

Muennig, P. (2008). The body politic: the relationship between stigma and obesity-associated disease. BMC Public Health 8, 128. doi: 10.1186/1471-2458-8-128

Neely, J.H. (1977). Semantic priming and retrieval from lexical memory: roles of inhibitionless spreading of activation and limited-capacity attention. J. Exp. Psychol. Gen. 106, 226-254.

Öhman, A., Lundqvist, D., and Esteves, F. (2001). The face in the crowd revisited: a threat advantage with schematic stimuli. J. Pers. Soc. Psychol. 80 381-396.

Potts, G. F., and Tucker, D. M. (2001) Frontal evaluation and posterior representation in target detection. Cogn. Brain Res. 11, 147-156.

Powdermaker, H. (1960). An anthropological approach to the problem of obesity. Bull. N. Y. Acad. Med. 36, 286-295.

Pudel, V., and Westenhöfer, J. (1989). Fragebogen zum Essverhalten (FEV). Göttingen: Verlag für Psychologie.

Puhl, R., and Brownell, K. D. (2001). Bias, discrimination, and obesity. Obes. Res. 9, 788-805.

Puhl, R. M., and Latner, J. D. (2007) Stigma, obesity, and the health of the nation's children. Psychol. Bull. 133 557-580.

Puhl, R. M., Schwartz, M. B., and Brownell, K. D. (2005). Impact of perceived consensus on stereotypes about obese people: a new approach for reducing bias. Health Psychol. 24, 517-525.

Sabbagh, M. A., and Taylor, M. (2000). Neural correlates of theory-of-mind reasoning: an event-related potential study. Psychol. Sci. 11, 46-50.

Schupp, H. T., Flaisch, T., Stockburger, J. and Junghofer, M. (2006). Emotion and attention: event-related brain potential studies. Prog. Brain Res. 156, 31-51.

Schupp, H. T., Öhman, A., Junghöfer, M., Weike, A. I., Stockburger, J., and Hamm, A. O. (2004). The facilitated processing of threatening faces: an ERP analysis. Emotion 4, 189-200.

Schupp, H. T., Stockburger, J., Codispoti, M., Junghöfer, M., Weike, A. I., and Hamm, A. O. (2007a). Selective visual attention to emotion. J. Neurosci. 27, 1082-1089.

Schupp, H. T., Stockburger, J., Bublatzky, F., Junghöfer, M., Weike, A. I., and Hamm, A. O. (2007b). Explicit attention interferes with selective emotion processing in human extrastriate cortex. BMC Neurosci. 8, 16. doi: 10.1186/1471-2202-8-16

Schwartz, M. B., Vartanian, L. R., Nosek, B. A., and Brownell, K. D. (2006). The influence of one's own body weight on implicit and explicit anti-fat bias. Obesity 14, 440-447.

Smid, H. G., Jakob, A., and Heinze, H. J. (1999). An event-related brain potential study of visual selective attention to conjunctions of color and shape. Psychophysiology 36, 264-279.

Snodgrass, J. G., and Vanderwart, M. (1980). Standardized set of 260 pictures - norms for name agreement, image agreement, familiarity, and visual complexity. J. Exp. Psychol. Hum. Learn. 6, 174-215.

Stockburger, J., Renner, B., Weike, A. I., Hamm, A. O., and Schupp, H. T. (2009). Vegetarianism and food perception. Selective visual attention to meat pictures. Appetite 52, 513-516.

Teachman, B. A., and Brownell, K. D. (2001). Implicit anti-fat bias among health professionals: is anyone immune? Int. J. Obes. 25, 1525-1531.

Teachman, B.A., Gapinski, K. D., Brownell, K. D., Rawlins, M., and Jeyaram, S. (2003). Demonstrations of implicit anti-fat bias: the impact of providing causal information and evoking empathy. Health Psychol. 22, 68-78.

Thompson, M. A., and Gray, J. J. (1995). Development and validation of a new body-image assessment scale. J. Pers. Assess. 64, 258-269.

Thorpe, S., Fize, D., and Marlot, C. (1996). Speed of processing in the human visual system. Nature 381, 520-522.

Todorov, A., Mandisodza, A. N., Goren, A., and Hall, C. C. (2005). Inferences of competence from faces predict election outcomes. Science 308, 1623-1626.

Todorov, A., Said, C. P., Engell, A. D., and Oosterhof, N. N. (2008). Understanding evaluation of faces on social dimensions. Trends Cogn. Sci. 12, 455-460.

Todorov, A., and Uleman, J.S. (2003). The efficiency of binding spontaneous trait inferences to actors' faces. J. Exp. Soc. Psychol. 39, 549-562.

Van Berkum, J. J., Holleman, B., Nieuwland, M., Otten, M., and Murre, J. (2009). Right or wrong? The brain's fast response to morally objectionable statements. Psychol. Sci. 20, 1092-1099.

Wang, S. S., Brownell, K. D., and Wadden, T. A. (2004). The influence of the stigma of obesity on overweight individuals. Int. J. Obes. 28, 1333-1337.

Wardle, J., Volz, C., and Golding, C. (1995). Social variation in attitudes to obesity in children. Int. J. Obes. 19, 562-569. 
Wiens, S., Sand, A., and Olofsson, J. K. (2011). Nonemotional features suppress early and enhance late emotional electrocortical responses to negative pictures. Biol. Psychol. 86, 83-89.

Wieser, M. J., Pauli, P., Reicherts, P., and Muhlberger, A. (2010). Don't look at me in anger! Enhanced processing of angry faces in anticipation of public speaking. Psychophysiology 47, 271-280.

Willis, J., and Todorov, A. (2006). First impressions: making up your mind after a $100-\mathrm{ms}$ exposure to a face. Psychol. Sci. 17, 592-598.

Conflict of Interest Statement: The authors declare that the research was conducted in the absence of any commercial or financial relationships that could be construed as a potential conflict of interest.

Received: 23 July 2010; accepted: 19 February 2011; published online: 04 March 2011.

Citation: Schupp HT and Renner B (2011) The implicit nature of the anti-fat bias.
Front. Hum. Neurosci. 5:23. doi: 10.3389/ fnhum.2011.00023

Copyright (c) 2011 Schupp and Renner. This is an open-access article subject to an exclusive license agreement between the authors and Frontiers Media SA, which permits unrestricted use, distribution, and reproduction in any medium, provided the original authors and source are credited. 